\title{
Finite-Set Model Predictive Control for Eight-Switch Three-Phase NPC Converter with DC-Link Voltages Fluctuation Suppression
}

\author{
Zeyu Wang ${ }^{1}$ and Molin Zhao ${ }^{2, *}$ \\ ${ }^{1}$ College of Electrical and Electronic Engineering, Shandong University of Technology, Shandong 255049, China \\ ${ }^{2}$ Department of Automation and Electrical Engineering, Yanshan University, QinHuangdao 066004, China \\ ${ }^{*}$ Corresponding author
}

\begin{abstract}
The three-level neutral point clamped converter (NPC) is one of the most promising topologies for medium-voltage high-power applications. However, the system stability is challenged by the DC-link capacitor voltages fluctuation especially in the presence of fault tolerant operation condition. To solve this, a novel finite-set model predictive control (FS-MPC) for eightswitch three-phase NPC converter (ESTP-NPC) in balanced grids is proposed for fault tolerant operation in this paper. The main objective of this work is to achieve the DC-link capacitor voltages fluctuation suppression without adding any additional components. Specifically, in order to keep the capacitor voltages balancing, an exact constraint term is introduced into the normalized cost function used in the ESTP-NPC. Thus, the system stability can be enhanced, and the DC-link capacitor voltages fluctuation can be suppressed effectively. Finally, simulation results are presented to verify the efficacy of the proposed method.
\end{abstract}

Keywords-finite-set model predictive control; eight-switch three-level neutral point clamped converter; cost function

\section{INTRODUCTION}

The three-level neutral point clamped converter (NPC) has been widely applied in industries over the past decades, such as electrical drives [1], high-voltage direct current (HVDC) transmission systems [2], and electric railway traction system [3], etc. In this topology, the bidirectional power flow is achieved based on charging and discharging of the DC-link capacitors [4]. Besides, compared to the traditional diode rectifier, it has following advantages: 1) low-harmonic distortion of grid-side currents; 2) unity power factor operation; 3) controlled DC-link output voltage. Due to these salient merits, numerous investigations are devoted toward the application of three-level NPC in balanced grids [5-6].

As the core component of the grid, the reliable and stable operation of three-level NPC is the basis of the whole system. However, the switching devices of three-level NPC are inevitably damaged during the grid fault [7]. In that sense, the power quality will be deteriorated, and the whole system has a destabilizing impact. Therefore, it is significant to investigate the control method of three-phase NPC especially in the presence of fault tolerant operation condition.

To solve this, an eight-switch three-phase NPC converter (ESTP-NPC) for fault tolerant operation is presented to enhance the system stability. Meanwhile, various control methods are proposed in [8-10]. In [8], a mathematical model of the three phase four-switch (TPFS) pulse width modulation (PWM) voltage-source rectifier (VSR) in rotating $d-q$ frame is firstly derived. The result relies on using a so-called "reduced Park Transformation" on the switching functions. Based on [8], the modeling, modulation, and control of the TPFS PWM rectifier under balanced voltage are presented by using space vector pulse width modulation (SVPWM) method in [9]. In this method, a capacitor voltage oscillation and deviation is considered and built in the $d-q$ synchronous frame to facilitate the controller design. Thus, a capacitor voltage balancing can be achieved. However, a pulse-width modulator is used in controller design in [8] and [9], and then, this will be further complicated in practical application. In order to avoid the using of pulse-width modulator, a novel control method based on finite control-set model predictive control (FCS-MPC) for fault tolerant operation is proposed to reduce harmonic currents and output power fluctuations under unbalanced grid voltages in [10]. Although numerical examples are investigated to show the effectiveness of the aforementioned control methods, there are barely any studies that address DC-link capacitor voltages fluctuation for ESTP-NPC especially in the presence of fault tolerant operation condition.

To solve this issue, in this paper, we aim to achieve the DClink capacitor voltages fluctuation suppression for ESTP-NPC without adding any additional components. Thus, a novel finiteset model predictive control (FS-MPC) for ESTP-NPC in balanced grids is proposed for fault tolerant operation. In this method, in order to keep the capacitor voltages balancing, an exact constraint term is introduced into the normalized cost function used in the ESTP-NPC. Thus, the system stability can be enhanced, and the DC-link capacitor voltages fluctuation can be suppressed effectively. Finally, simulation results are presented to verify the efficacy of the proposed FS-MPC method.

\section{FCS-MPC METHOD OF ESTP-NPC}

The common topology of the ESTP-NPC is shown in Figure I. The ESTP-NPC with eight switches is connected to the threephase grid-side voltages through the grid-side input inductances $L_{g}$ and input resistances $R_{g}$. 


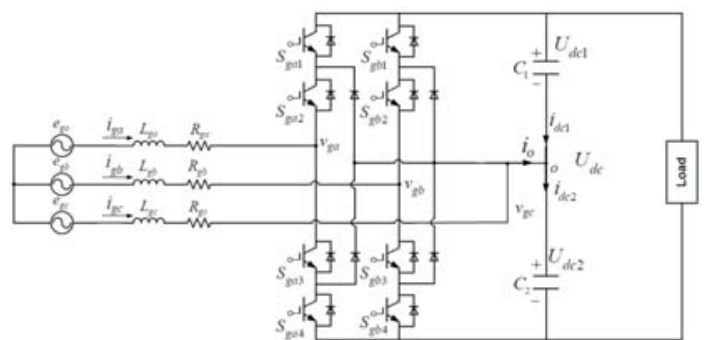

FIGURE I. THE COMMON TOPOLOGY OF THE ESTP-NPC

Considering all the possible combinations of $\left(S_{a}, S_{b}\right)$, there are eight output voltage vectors generated, and the corresponding vector positions are given in Figure II. From Figure II, the ESTP-NPC output voltage vector can be obtained to calculate the switching state vector and DC-link voltage as follows.

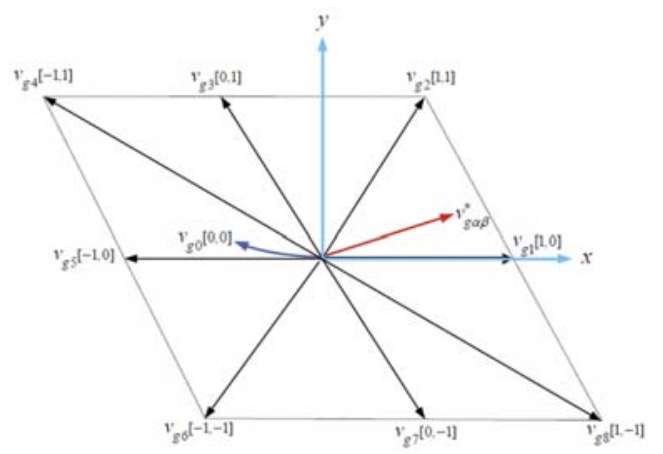

FIGURE II. VECTOR POSITIONS IN THE $\alpha-\beta$ PLANE OF THE FSTPGCCS.

$$
v_{g x}=S_{g x} \frac{U_{d c}}{2}
$$

Where $U_{d c}$ is the DC-link voltage, $x=a, b, c$, and the switching function $S_{g x}$ is defined according to the switching signals for the switches of each leg as follows:

$$
S_{g x}=\left\{\begin{array}{l}
1, S_{g \times 1}=\text { on }, S_{g \times 2}=\text { on, } S_{g \times 3}=\text { off }, S_{g \times 4}=\text { off } \\
0, S_{g \times 1}=\text { on, } S_{g \times 2}=\text { off }, S_{g \times 3}=\text { off }, S_{g \times 4}=\text { on } \\
-1, S_{g \times 1}=\text { off }, S_{g \times 2}=\text { off }, S_{g \times 3}=\text { on }, S_{g \times 4}=\text { on }
\end{array}\right.
$$

The grid-side current dynamics of the ESTP-NPC can be presented as

$$
L_{g} \frac{d i_{g}}{d t}=e_{g}-v_{g}-R_{g} i_{g}
$$

Based on the forward Euler approximation method, the predicted grid-side current can be obtained as follows.

$$
i_{g}(k+1)=\left(1-\frac{R_{g} T_{s}}{L_{g}}\right) i_{g}(k)+\frac{T_{s}}{L_{g}}\left[e_{g}(k)-v_{g}(k)\right] .
$$

The block diagram of the proposed FS-MPC for ESTPNPC is shown in Figure III. From Figure III, the expression of active power and reactive power in the stationary reference frame $\alpha-\beta$ can be expressed by [11]:

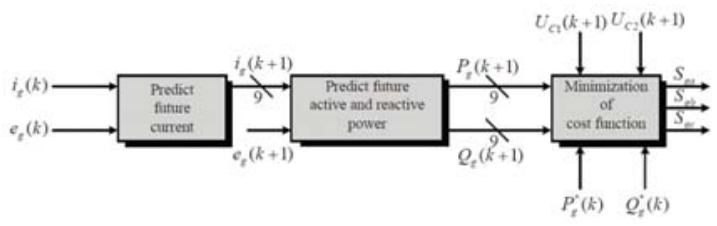

FIGURE III. BLOCK DIAGRAM OF THE PROPOSED FS-MPC FOR ESTP-NPC

$$
\begin{aligned}
P_{g}(k+1) & =\operatorname{Re}\left\{e_{g}(k+1) \bar{i}_{g}(k+1)\right\} \\
& =\left(e_{g \alpha} i_{g \alpha}+e_{g \beta} i_{g \beta}\right), \\
Q_{g}(k+1) & =\operatorname{Im}\left\{e_{g}(k+1) \bar{i}_{g}(k+1)\right\} \\
& =\left(e_{g \beta} i_{g \alpha}-e_{g \alpha} i_{g \beta}\right),
\end{aligned}
$$

Where $e_{g}(k+1)$ is the predicted grid-side voltage.

Then, a cost function is defined as a criterion to select the optimal switching states

$$
f_{1}=\left|P_{g}^{*}-P_{g}(k+1)\right|^{2}+\left|Q_{g}^{*}-Q_{g}(k+1)\right|^{2},
$$

Where $P_{g}(k+1)$ and $Q_{g}(k+1)$ are the predicted active and reactive power, $P_{g}^{*}$ and $Q_{g}^{*}$ are the reference active and reactive power.

\section{The Proposed FS-MPC METHOD WITH DC-LINK VOLTAGES FLUCTUATION SUPPRESSION}

In traditional FCS-MPC method, the capacitor voltage oscillations and deviations will result in a destabilizing impact [12-13]. To resolve this defect, a novel FS-MPC for ESTP-NPC in balanced grids is proposed for fault tolerant operation as follows. More specifically, in order to keep the capacitor voltages balancing, an exact constraint term is introduced into the normalized cost function used in the ESTP-NPC.

The continuous-time model of capacitor voltages can be expressed as follows:

$$
\left\{\begin{array}{l}
U_{C 1}(t)=U_{C 1}(0)+\frac{1}{\mathrm{C}_{1}} \int_{0+}^{t} i_{C 1}(\tau) d \tau, \\
U_{C 2}(t)=U_{C 2}(0)+\frac{1}{\mathrm{C}_{2}} \int_{0+}^{t} i_{C 2}(\tau) d \tau,
\end{array}\right.
$$


Where $U_{C x}(0)(x=1,2)$ represents the initial value of capacitor voltages, and $i_{C x}(x=1,2)$ represents the current flowing through the capacitors.

The discrete form of (8) can be obtained as

$$
\left\{\begin{array}{l}
U_{C 1}(k+1)=U_{C 1}(k)+\frac{T_{s}}{C_{1}} i_{C 1}(k), \\
U_{C 2}(k+1)=U_{C 2}(k)+\frac{T_{s}}{C_{2}} i_{C 2}(k),
\end{array}\right.
$$

Where $C_{x}(x=1,2)$ represents the capacitor, and $T_{s}$ represents the sampling period.

Thus, the cost function can be redefined to achieve the capacitor voltages balancing control.

$$
\begin{aligned}
f_{2} & =\left|P_{g}^{*}-P_{g}(k+1)\right|^{2}+\left|Q_{g}^{*}-Q_{g}(k+1)\right|^{2} \\
& +\lambda_{d}\left|U_{C 1}(k+1)-U_{C 2}(k+1)\right|^{2},
\end{aligned}
$$

Where $\lambda_{d}$ represents the weighting factor for DC-link capacitor voltages balancing control. In this paper, an empirical tuning method is adopted to obtain the appropriate weighting factor [14-15]. Besides, the cost function given in (10) is evaluated for all available switching states. The optimal switching state which minimizes the cost function is selected and applied to the ESTP-NPC. Thus, the capacitor voltage balancing can be achieve, and the system performance can be enhanced. In order to verify the efficacy of the proposed scheme, the system simulation results will be given as follows.

\section{Simulation REsults}

The system simulations are implemented in MATLAB/Simulink environment. The system parameters are given in Table I.

TABLE I. SYSTEM PARAMETERS

\begin{tabular}{ccc}
\hline input resistance & $R_{g}$ & $0.01 \Omega$ \\
input inductance & $L_{g}$ & $11 \mathrm{mH}$ \\
DC-link capacitor & $C_{1} / C_{2}$ & $4400 \mu \mathrm{F}$ \\
load resistance & $R_{\text {Load }}$ & $50 \Omega$ \\
phase voltage peak & $e_{g}$ & $100 \mathrm{~V}$ \\
grid frequency & $f$ & $50 \mathrm{~Hz}$ \\
DC-link reference voltage & $U_{d c}$ & $500 \mathrm{~V} / 550 \mathrm{~V}$ \\
sampling period & $T_{s}$ & $30 \mu \mathrm{s}$ \\
weighting factor & $\lambda_{d c}$ & 0.3 \\
PI controller & $K_{p}=0.04$ & $K_{i}=0.38$
\end{tabular}

To further evaluate the steady-state and transient-state performances of the proposed FS-MPC method for the ESTPNPC, the simulation results are presented in Figure IV - Figure
V. As shown in Figure IV(a) and Figure V(a), they show that the active/reactive power of the ESTP-NPC is able to track the reference value accurately. The phase-a grid-side current is always in phase with phase-a grid-side voltage during the steady-state and transient-state, and shown in Figure IV(b) and Figure V(b). Noted that the THD of grid current is $2.25 \%$ before the step $t=2.5 \mathrm{~s}$ to $2.00 \%$ after the step in DC-link voltage reference. Besides, as shown in Figure IV(c) and Figure $\mathrm{V}(\mathrm{c})$, it can be clearly seen that the DC-link voltage performs well without any overshoot or undershoot. The capacitor voltage waveforms are presented in Figure IV(d) and Figure V(d). From aforementioned results it can be concluded that the effectiveness of the proposed FS-MPC method with DC-link capacitor voltages fluctuation suppression can be confirmed in the steady-state and transient-state.
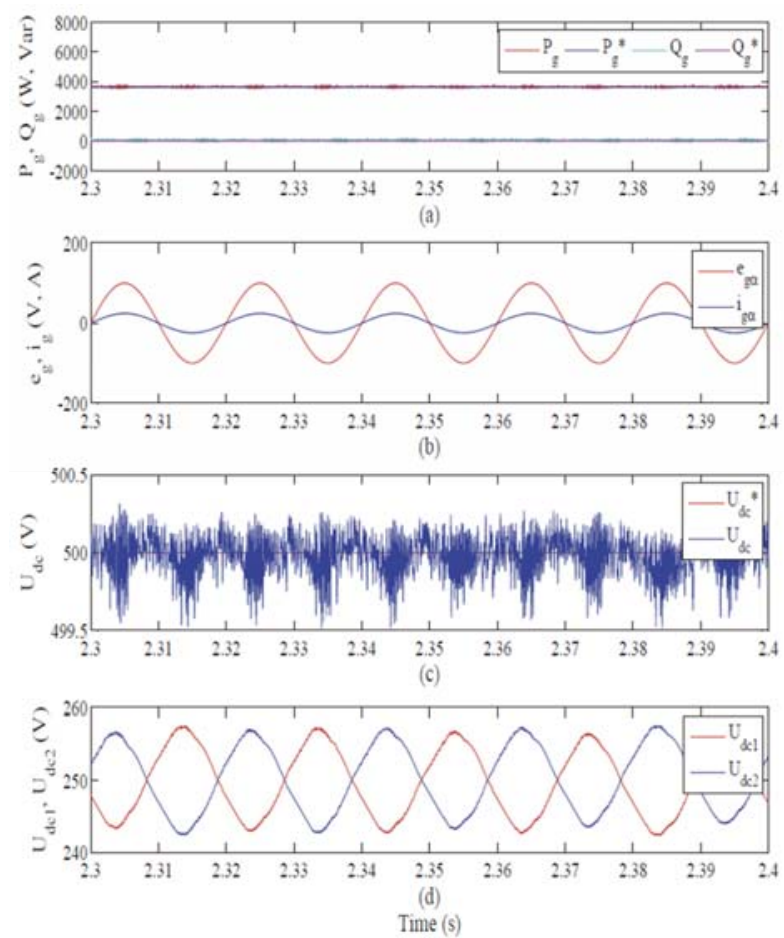

FIGURE IV. SIMULATION RESULTS: STEADY-STATE PERFORMANCE OF PROPOSED FS-MPC FOR THE ESTP-NPC (a) GRID-SIDE POWERS (b) GRID-SIDE VOLTAGE AND CURRENT (c) DC-LINK VOLTAGE (d) DC-LINK CAPACITOR VOLTAGE 

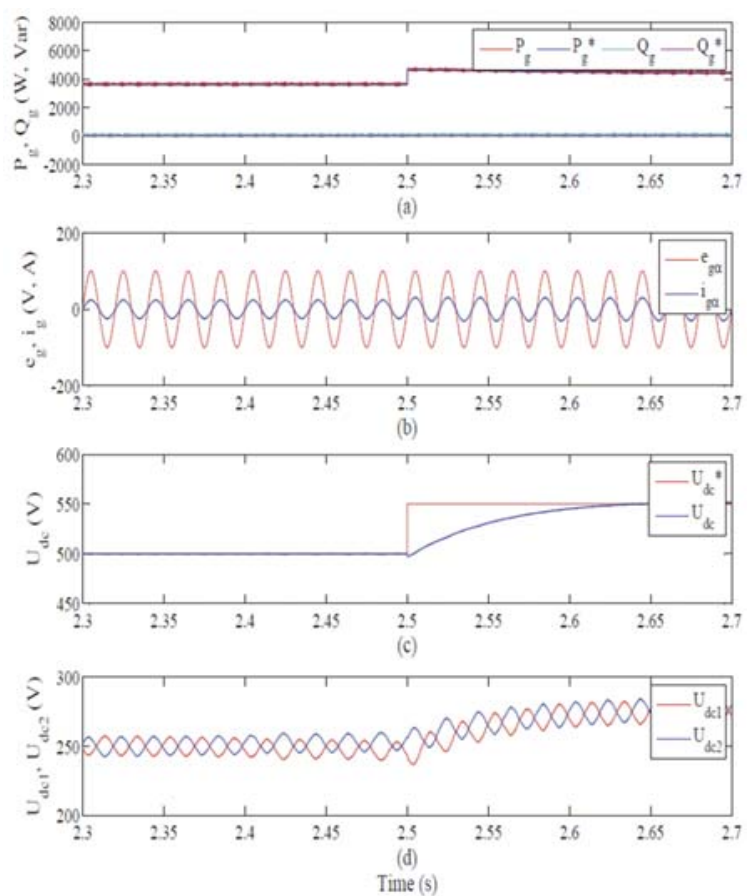

FIGURE V. SIMULATION RESULTS: TRANSIENT-STATE

PERFORMANCE OF PROPOSED FS-MPC FOR THE ESTP-NPC (a) GRID-SIDE POWERS (b) GRID-SIDE VOLTAGE AND CURRENT

(c) DC-LINK VOLTAGE (d) DC-LINK CAPACITOR VOLTAGE

\section{CONCLUSIONS}

In this paper, an improved FS-MPC method with DC-link voltages offset suppression for the ESTP-NPC is presented by using a single step prediction horizon. In the proposed method, the ESTP-NPC fault tolerant structure is reconstructed from the conventional three-level NPC for medium-voltage, high-power applications. Meanwhile, in order to keep the capacitor voltages balancing, an exact constraint term is introduced into the normalized cost function used in the ESTP-NPC. Thus, the system stability can be enhanced, and the DC-link capacitor voltages fluctuation can be suppressed effectively. The results confirm that the efficacy of the proposed method.

\section{REFERENCES}

[1] Habibullah M, Lu D C, Xiao D, et al. Predictive Torque Control of Induction Motor Sensorless Drive Fed by a 3L-NPC Inverter[J]. IEEE Transactions on Industrial Informatics, 2017, 13(1):60-70.

[2] Calle-Prado A, Alepuz S, Bordonau J, et al. Model Predictive Current Control of Grid-Connected Neutral-Point-Clamped Converters to Meet Low-Voltage Ride-Through Requirements[J]. IEEE Transactions on Industrial Electronics, 2015, 62(3):1503-1514.

[3] Hu J, Zhu J, Dorrell D G. Model Predictive Control of Grid-Connected Inverters for PV Systems With Flexible Power Regulation and Switching Frequency Reduction[J]. IEEE Transactions on Industry Applications, 2013, 51(1):587-594.

[4] Zhang Z, Hackl C, Kennel R. Computationally Efficient DMPC for Threelevel NPC Back-to-Back Converters in Wind Turbine Systems With PMSG[J]. IEEE Transactions on Power Electronics, 2016, 32(10):80188034.

[5] Calle-Prado A, Alepuz S, Bordonau J, et al. Predictive Control of a Backto-Back NPC Converter-Based Wind Power System[J]. IEEE Transactions on Industrial Electronics, 2016, 63(7):4615-4627.

[6] Zhang Z, Wang F, Sun T, et al. FPGA-Based Experimental Investigation of a Quasi-Centralized Model Predictive Control for Back-to-Back Converters[J]. IEEE Transactions on Power Electronics, 2015, 31(1):662674.

[7] Liu Y C, Ge X, Tang Q, et al. Two modified SVPWM algorithms for common-mode voltage reduction in eight-switch three-phase inverters[J]. Electronics Letters, 2017, 53(10):676-678.

[8] Lee T S, Liu J H. Modeling and Control of a Three-Phase Four-Switch PWM Voltage-Source Rectifier in d-q Synchronous Frame[J]. IEEE Transactions on Power Electronics, 2011, 26(9):2476-2489.

[9] Zeng Z, Zheng W, Zhao R, et al. Modeling, Modulation, and Control of the Three-Phase Four-Switch PWM Rectifier Under Balanced Voltage[J]. IEEE Transactions on Power Electronics, 2016, 31(7):4892-4905.

[10] Jin N, Hu S, Gan C, et al. Finite States Model Predictive Control for Fault Tolerant Operation of Three-Phase Bidirectional AC/DC Converter Under Unbalanced Grid Voltages[J]. IEEE Transactions on Industrial Electronics, 2018, 65(1):819-829.

[11] Peng F Z, Lai J S. Generalized instantaneous reactive power theory for three-phase power systems[J]. IEEE Transactions on Instrumentation and Measurement, 1996, 45(1):293-297.

[12] Akter M P, Lu D C, Zhu J. Model predictive controlled three-level activeneutral-point-clamped inverter with improved computational speed and stability, and balanced DC-link voltages[C]. Future Energy Electronics Conference and Ecce Asia. IEEE, 2017:116-121.

[13] Vargas R, Cortes P, Ammann U, et al. Predictive Control of a Three-Phase Neutral-Point-Clamped Inverter[J]. IEEE Transactions on Industrial Electronics, 2007, 54(5):2697-2705.

[14] Rodriguez J, Cortes P, Kennel R, et al. Model Predictive Control-A Simple and Powerful Method to Control Power Converters[J]. IEEE Transactions on Industrial Electronics, 2009, 56(6):1826-1838.

[15] Rodriguez J, Kazmierkowski M P, Espinoza J R, et al. State of the Art of Finite Control Set Model Predictive Control in Power Electronics[J]. IEEE Transactions on Industrial Informatics, 2013, 9(2):1003-1016. 